\title{
Osteoporosis in Inflammatory Bowel Disease
}

\author{
Tauseef Ali, MD ${ }^{a}, b$, David Lam, MD ${ }^{a}$, Michael S. Bronze, MDa ${ }^{a}$, and Mary Beth Humphrey, MD, \\ PhDa,c \\ aDepartment of Medicine, University of Oklahoma Health Sciences Center, Oklahoma City. \\ bSection of Digestive Diseases and Nutrition, University of Oklahoma Health Sciences Center, \\ Oklahoma City. \\ 'Section of Rheumatology, Immunology and Allergy, University of Oklahoma Health Sciences \\ Center, Oklahoma City.
}

\begin{abstract}
Osteoporosis commonly afflicts patients with inflammatory bowel disease, and many factors link the 2 states together. A literature review was conducted about the pathophysiology of osteoporosis in relation to inflammatory bowel disease. Screening guidelines for osteoporosis in general as well as those directed at patients with inflammatory bowel disease are reviewed, as are currently available treatment options. The purpose of this article is to increase physician awareness about osteopenia and osteoporosis occurring in patients with inflammatory bowel disease and to provide basic, clinically relevant information about the pathophysiology and guidelines to help them treat these patients in a cost-effective manner.
\end{abstract}

\section{Keywords}

Inflammatory bowel disease; Osteopenia; Osteoporosis

Approximately 1.4 million people in the United States and 2.2 million people in Europe suffer from inflammatory bowel disease. ${ }^{1}$ Patients with inflammatory bowel disease are at higher risk of developing osteoporosis and osteopenia than the general population, with relative risk of fracture $40 \%$ higher in patients with inflammatory bowel disease. ${ }^{2}$ The prevalence of osteopenia and osteoporosis in patients with inflammatory bowel disease varies significantly depending on the study populations, location, and design, but ranges from $22 \%-77 \%$ and $17 \%-41 \%$, respectively. ${ }^{3,4}$ Because of the high prevalence, it is imperative that physicians recognize patients at risk for osteoporosis, screen appropriate patients, and prevent or treat accordingly.

\section{PATHOPHYSIOLOGY OF OSTEOPOROSIS IN INFLAMMATORY BOWEL DISEASE}

Bone is a living tissue that undergoes constant remodeling by bone-forming cells (osteoblasts) and bone-resorbing cells (osteoclasts). Imbalances in bone formation and resorption lead to osteoporosis. Several gastrointestinal dis orders have been associated with osteoporosis and

Requests for reprints should be addressed to Mary Beth Humphrey, MD, PhD, Department of Medicine, University of Oklahoma Health Sciences Center, 975 N.E. $10^{\text {th }}$ St., BRC 209, Oklahoma City, OK 73104. marybeth-humphrey @ ouhsc.edu.

Conflict of Interest: None.

Authorship: All authors contributed to writing this manuscript. 
osteopenia, including inflammatory bowel disease, celiac disease, and chronic liver disease.

5,6 The etiology of osteoporosis in inflammatory bowel disease is multifactorial, with risk

factors including age, corticosteroid use, malnutrition, vitamin D and calcium malabsorption and deficiency, immobilization, and the underlying inflammatory state (Table 1). ${ }^{5}$

\section{ROLE OF CORTICOSTEROIDS}

Because glucocorticoids are a treatment mainstay for chronic inflammatory diseases, it is important to recognize the effects they have on bone remodeling. They have been shown to impair osteoblast function, induce osteoblast apoptosis, reduce intestinal calcium absorption, and increase renal excretion of calcium. ${ }^{5,7}$ Patients on glucocorticoids are at increased risk for fracture, with the greatest bone loss occurring in the initial months of treatment. ${ }^{8}$ Interestingly, studies show a decrease in fracture risk back toward baseline after stopping glucocorticoid therapy. ${ }^{9}$ It is, however, difficult to distinguish how much impact glucocorticoid use has on bone compared with disease activity, as increased disease activity and higher degrees of inflammation are indications for steroid use. While prednisone, prednisolone, and methylprednisolone are systemically acting steroids and one of the major contributing factors in osteoporosis in inflammatory bowel disease, budesonide, a locally acting corticosteroid with low systemic bioavailability, has been increasingly used in the treatment of inflammatory bowel disease because of its lack of systemic effects, including prevention of bone loss.

\section{ROLE OF INFLAMMATION}

There is a developing wealth of information about the role of inflammation in Crohn's disease and ulcerative colitis and the development of osteoporosis. In inflammatory bowel disease, the immune response, mediated by $\mathrm{T}$ lymphocytes and other inflammatory cells like macrophages, leads to production of various proinflammatory cytokines such as interleukin (IL)-2 and tumor necrosis factor (TNF). Within mononuclear cells, the key nuclear transcription factor is nuclear factor-kappa $\mathrm{B}(\mathrm{NF} \kappa \mathrm{B})$, which regulates the transcription of IL-1, IL-6, IL-8, and other peptides central to the inflammatory response. ${ }^{10}$ Additionally $\mathrm{NF} \kappa \mathrm{B}$ regulates expression of other proinflammatory genes such as TNF- $\alpha$, adhesion molecules, and chemokines. ${ }^{11}$

Levels of many proinflammatory osteoclast activators including TNF- $\alpha$, interleukins $1 \alpha$ and $1 \beta$ (IL-1 $\alpha$, IL-1 $\beta$ ), IL-6, IL-11, IL-17, transforming growth factor alpha, epidermal growth factor, and prostaglandin $E_{2}$ are increased in patients with inflammatory bowel disease. $5,6,12$ Evidence supports IL-6 to be a pathogenic factor in osteoporosis that results from the loss of either male or female sex steroids, and studies have implicated IL-6 in the pathophysiology of several other diseases, such as rheumatoid arthritis. ${ }^{13}$ Genetic variations in the IL-6 and IL-1 receptor antagonist genes are correlated with the clinical course of inflammatory bowel disease and extent of bone loss. ${ }^{14}$

A new TNF family pathway involved in bone metabolism, known as the RANK-RANKL-OPG pathway, has been recently described. ${ }^{15}$ RANKL (receptor activator of NF- $\kappa$ B ligand) is expressed on the surface of osteoblasts, synovial stromal cells, and activated T cells. RANKL binds to either osteoclast precursors expressing the RANKL receptor, RANK (receptor activator of NF- $\kappa \mathrm{B}$ ) or a soluble decoy receptor osteoprotegerin (OPG), which is produced by osteoblasts. If RANKL and RANK interact, osteoclasts differentiate and mature, resulting in increased bone loss. OPG blocks this interaction, thereby inhibiting osteoclast production. Compounds such as parathyroid hormone, $1 \alpha, 25-(\mathrm{OH})_{2} \mathrm{D}_{3}$, prostaglandin $\mathrm{E}_{2}$, and dexamethasone stimulate RANKL expression and inhibit OPG production, thereby causing increased osteoclastogenesis, whereas $17 \beta$-estradiol increases OPG and decreases RANKL, reducing osteoclastogenesis. ${ }^{15}$ With this information, the RANK-RANKL-OPG system might be the final common pathway accounting for the activity of many of the compounds known to act on bone homeostasis. 
Recent studies have suggested that alterations in RANKL: OPG ratios might be responsible for bone loss in patients with inflammatory bowel disease. Plasma levels of OPG and RANKL are correlated with bone mineral density and current inflammatory bowel disease therapy. ${ }^{16}$ In one study, OPG plasma levels were found to be elevated 2.4-fold in Crohn's disease and 1.9-fold in ulcerative colitis. ${ }^{17}$ The increased OPG levels may represent a continuing homeostatic response, attempting to reverse RANKL or TNF- $\alpha$-driven osteoclastogenesis, thus attempting to maintain normal bone mass. Osteoclastogenic factors released from the inflamed mucosa, including IL-1, IL-6, and TNF- $\alpha$ may drive osteoclastogenesis indirectly by enhancing RANKL expression on stromal osteoblastic cells. However, IL- $1 \beta$ and TNF- $\alpha$ concomitantly increase OPG expression by stromal osteoblasts. ${ }^{18}$ Thus, cytokines known to perpetuate colitis simultaneously alter the RANKL:OPG ratio.

Therapy with anti-TNF- $\alpha$ drugs such as infliximab improves bone mineral density and alters serum markers of bone remodeling. ${ }^{19-21}$ Osteocalcin, a marker of bone formation, increases, while beta-cross-links, a bone resorption marker, decreases after infliximab treatment. ${ }^{19}$

Furthermore, OPG decreases after therapy, indicative of its counter-regulatory role relative to inflammatory cytokines. ${ }^{19}$ Longitudinal studies of OPG/RANKL and bone mineral density are required to validate this and to assess the relative contribution of inflammation to bone loss in human disease.

\section{CLINICAL SIGNIFICANCE}

- Patients with inflammatory bowel disease are at high risk of developing osteoporosis and osteopenia.

- Risk factors in this population include disease activity, corticosteroid use, malnutrition, and deficiencies in vita-min $\mathrm{D}$ or calcium.

- Based on screening guidelines, patients at risk should have a screening bone mineral density.

- Both pharmacologic and nonpharmacologic therapy should be used for treatment.

\section{GENETIC FACTORS}

The recent discoveries of signal transduction pathways and transcription factors critical for osteoblast differentiation and function have opened up new approaches to the understanding of the pathogenesis of osteoporosis. ${ }^{22}$ Identification of the critical role for the Wnt (wingless genes) signaling pathway in regulating osteoblast function is of particular interest, because it has been shown to play an important role in determining bone mass and strength. ${ }^{23}$ The precise mechanisms whereby Wnt signaling alters osteoblast function are not fully understood, but there is evidence that the canonical $\beta$-catenin pathway is involved and that there is an interaction with bone morphogenetic protein $2 .{ }^{24}$ The inhibition of skeletal growth by glucocorticoids might be mediated by effects on Wnt signaling. ${ }^{25}$

The identification of vitamin D receptors in peripheral blood mononuclear cells sparked the early interest in vitamin D as an immune system regulator. ${ }^{26}$ Vitamin D deficiency has been linked to several different diseases, including the immune system-mediated osteoporosis such as inflammatory bowel disease. The potential role of vitamin D deficiency in patients with inflammatory bowel disease and osteoporosis is further discussed in the section on nutritional factors. 


\section{NUTRITIONAL FACTORS}

Inflammatory bowel disease-related nutritional deficiencies have been implicated as other pathogenic mechanisms resulting in low bone mineral density. Calcium is required for normal growth and development of the skeleton. Adequate calcium intake is critical to achieving optimal peak bone mass and modifies the rate of bone loss associated with aging. Calcium deficiency (as a result of either low intake or poor intestinal absorption) has been reported in Crohn's disease. ${ }^{27}$ Vitamin D deficiency has been reported more frequently in patients with ulcerative colitis and Crohn's disease compared with either control subjects or a healthy population reference range. ${ }^{28}$ The lower vitamin $\mathrm{D}$ status in patients with Crohn's disease compared with controls is accompanied by elevated levels of markers of bone turnover. ${ }^{29}$ Several reasons have been suggested for the lower vitamin $\mathrm{D}$ status of patients with inflammatory bowel disease, including a reduced efficiency of intestinal absorption of vitamin $\mathrm{D}$ as a consequence of ileopathy, a disrupted enterohepatic circulation of vitamin $\mathrm{D}$, renal insufficiency, reduced dietary intake, and reduced exposure to sunshine. ${ }^{30}$

Vitamin K deficiency also might be involved in inflammatory bowel disease-associated osteopenia. Patients with longstanding Crohn's disease, in remission and receiving no or very low doses of steroids, have low serum vitamin K concentrations and increased concentrations of uncarboxylated osteocalcin, ${ }^{31}$ a biochemical index of vitamin $\mathrm{K}$ status, which also predicts hip fracture risk. ${ }^{32}$ The reasons for the lower vitamin K status in patients with inflammatory bowel disease are unclear. Some patients may malabsorb this fat-soluble vitamin because of ileopathy. However, there also is the intriguing possibility that differences in vitamin $\mathrm{K}$ status in patients with ulcerative colitis (as well as patients with predominantly colonic Crohn's disease) may arise from altered bacterial flora that produce less vitamin K. ${ }^{28}$ It also is possible that antibiotics often used to treat patients with inflammatory bowel disease could kill vitamin K-producing flora. ${ }^{28}$ Clearly, the underlying reasons for low vitamin $\mathrm{K}$ status in patients with inflammatory bowel disease require further investigation.

\section{SCREENING GUIDELINES}

The World Health Organization (WHO) defines osteoporosis as bone mineral density at the hip or spine $<2.5$ standard deviations below the mean for young healthy sex- and race-matched adults. Although bone mineral density assessment is the most common means of diagnosing osteoporosis, the WHO has developed a web-based interactive tool, FRAX ${ }^{\mathrm{TM}}$ (Fracture Risk Assessment Model), that includes 10 risk factors. ${ }^{33}$ This tool helps physicians calculate the 10 -year probability of having major osteoporotic fractures, even in the absence of knowing the patient's actual bone mineral density. While bone mineral density is a major risk factor for fracture, this tool highlights the importance of other risk factors.

The American Gastroenterological Association (AGA) has published guidelines that recommend DXA (dual energy X-ray absorptiometry) screening in inflammatory bowel disease patients with one or more risk factors: history of vertebral fractures, postmenopausal, male $>50$ years of age, chronic corticosteroid therapy, or hypogonadism. ${ }^{6}$ If the initial DXA is normal, the AGA recommends repeat testing in 2-3 years. If the patient has osteoporosis, or has a history of a low trauma fracture, evaluation for secondary causes should be completed. Suggested studies include a complete blood count, serum concentrations of alkaline phosphatase level, calcium, creatinine, and $25-\mathrm{OH}$ vitamin $\mathrm{D}$, serum protein electrophoresis, serum calcium, and a testosterone level in males.

Utilization of these guidelines increases the number of screened patients and should lead to earlier diagnosis and treatment. Kornbluth et al showed that in 100 consecutive patients that met the AGA criteria for initial DXA, osteoporosis was found in $12 \%$, and osteopenia in another $44 \% .{ }^{34}$ Pharmacologic therapy was initiated in $89 \%$ of these patients, with 69 patients receiving 
calcium and vitamin $\mathrm{D}$, and 20 patients receiving bisphosphonates. Although this is a small and limited study, it shows how following guidelines led to interventions that might ultimately reduce fracture risk.

\section{TREATMENT OPTIONS}

\section{Nonpharmacologic Therapies}

Several nonpharmacologic therapies are recommended for all patients at risk for developing osteoporosis, including those suffering from inflammatory bowel disease (Table 2). ${ }^{6,35,36}$ These include regular weight-bearing exercise (resulting in improved bone mineral density and decreased risk of fall from increased agility, strength, and balance), avoidance of tobacco use and limiting alcohol intake (both risk factors on the WHO's Fracture Risk Assessment Tool), 33 and fall prevention. It is important that both patients and physicians recognize the risk factors for falls, as many of these can be easily corrected.

\section{Pharmacologic Therapies}

Calcium and Vitamin D-The AGA and British Society of Gastroenterology (BSG) recommend that patients at risk for or with osteoporosis receive calcium and vitamin D supplementation. The recommendation for younger men and premenopausal women is daily intake of elemental calcium of $1000 \mathrm{mg}$, either from diet or supplementation. Men and women over 50 years of age require $1500 \mathrm{mg}$ of calcium. Vitamin D intake of 400-800 IU/day should be adequate for relatively healthy individuals, but patients with intestinal malabsorption, chronic renal insufficiency, or housebound patients may need an increased amount. Supplementation levels of 25 -hydroxyvitamin $\mathrm{D}$ to $\geq 30 \mathrm{ng} / \mathrm{mL}$ or $75 \mathrm{nmol} / \mathrm{L}$ are preferred for patients with vitamin D deficiency. ${ }^{33}$

Bisphosphonates-Bisphosphonates have been FDA (Food and Drug Administration) approved for the prevention and treatment of osteoporosis in patients with known osteoporosis, atraumatic fractures, and patients who cannot withdraw from corticosteroids after 3 months of use. If patients are on corticosteroids for $>3$ months or deemed high risk based on other risk factors, the BSG recommends DXA first, and then consider bisphosphonate therapy if the Tscore is $<1.5 .^{36}$ The BSG also recommends bisphosphonate therapy at the onset of steroid therapy if the patient is $>65$ years old. A double-blind trial of 61 inflammatory bowel disease patients randomized to either 12 months of risedronate $5 \mathrm{mg}$ daily or placebo (all received calcium carbonate $600 \mathrm{mg}$ daily) showed a $2.0 \%$ and $1.9 \%$ improvement in bone mineral density at the spine and hip, respectively. ${ }^{37}$ In a prospective, open-label, 2-year extension study of a randomized, double-blind, 1 year clinical trial, 81 osteoporotic women with inflammatory bowel disease were treated with risedronate or placebo. ${ }^{38}$ Significant differences in lumbar spine, trochanter, and femoral neck bone mineral density were found between these 2 groups at 1,2, and 3 years. Importantly, the cumulative risk of vertebral and nonvertebral fractures was significantly lower in the risedronate group than in the placebo group. These data suggest that bisphosphonates are safe and effective in patients with inflammatory bowel disease.

Calcitonin-Salmon calcitonin (given intranasally, intramuscularly, or subcutaneously) is FDA approved for the treatment of osteoporosis in women who are at least 5 years postmenopausal. The Prevent Recurrence of Osteoporotic Fractures study demonstrated a 33\% reduction in the risk of new vertebral fractures in postmenopausal women with osteoporosis given salmon calcitonin intranasally at $200 \mathrm{IU} /$ day as compared with placebo. ${ }^{39}$ However, calcitonin is considered a second-line agent by the AGA and BSG because of cost and concerns for tachyphylaxis. It can rarely cause anaphylaxis, but more commonly, some patients experience rhinitis and epistaxis with the intranasal formulation. 
Hormone Therapy-Estrogen therapy is FDA approved for the prevention of osteoporosis in postmenopausal women or hypogonadal premenopausal women, but the risks and benefits must be weighed before initiating therapy. The Women's Health Initiative found that 5 years of hormone therapy reduced the risk of hip and vertebral fractures by $34 \%$, while reducing the risk of other osteoporotic fractures by $23 \% .{ }^{40}$ However, the Women's Health Initiative also showed increased risk of coronary artery disease, stroke, breast cancer, pulmonary embolism, and deep vein thrombosis with estrogen and progestin therapy. ${ }^{40}$ In light of this, it is recommended that estrogen replacement therapy be used at the lowest effective dose and for the shortest duration possible.

The selective estrogen receptor modulator, raloxifene, also has been approved for both prevention and treatment of osteoporosis in postmenopausal women. In a study of raloxifene versus placebo, the mean difference of bone mineral density was $2.4 \pm 0.4 \%$ at the lumbar spine, $2.4 \pm 0.4 \%$ for the total hip, and $2.0 \pm 0.4 \%$ for the total body at 24 months of therapy. ${ }^{41}$ Another study showed a $30 \%-50 \%$ risk reduction in vertebral fractures over 36 months. ${ }^{42}$ However, side effects include increased risk of deep vein thrombosis, hot flashes, and leg cramps. The AGA recommends that a bone disease specialist participate in the decision to utilize a selective estrogen receptor modulator in patients with gastrointestinal disease, and the BSG recommends their use in postmenopausal women who have either intolerance of or failure with bisphosphonates.

Parathyroid Hormone-Parathyroid hormone (1-34), or teriparatide, is approved for the treatment of osteoporosis in postmenopausal women at high risk of fracture and in men with primary or hypogonadal osteoporosis. It is administered as a subcutaneous injection for a maximum of 2 years. In a large, multicenter, placebo-controlled trial, recombinant parathyroid hormone produced a marked increase in bone mineral density, and decreased the risk of new vertebral fractures by $65 \%$ and nonvertebral fractures by $53 \% .{ }^{43}$ It is common to follow the 2year treatment period with another anti-resorptive agent, usually a bisphosphonate, to either maintain or further increase bone mineral density. While teriparatide is well tolerated in most patients, patients with a history of Paget's disease of the bone, prior skeletal radiation, prior skeletal malignancy (including bone metastases), or those with hypercalcemia should not be given teriparatide due to an increased risk of osteosarcoma (Black Box warning).

Corticosteroids and Immunomodulating Agents-While systemically acting steroids are a major contributing factor in osteoporosis in inflammatory bowel disease, a locally acting corticosteroid, budesonide, has been increasingly utilized in the treatment of both Crohn's disease and ulcerative colitis because of its lack of systemic effects. A recent case-control study looked at budesonide and different formulations of other orally administered steroids, including prednisone/prednisolone, methylprednisolone, and hydrocortisone. ${ }^{44}$ Oral prednisone/ prednisolone was associated with a dose-dependent increase in fracture risk starting from a dose of around $6.7 \mathrm{mg} / \mathrm{day}$. Oral budesonide and hydrocortisone were not associated with an increase in overall fracture risk. Another multi-center randomized trial of 272 patients found that treatment with budesonide compared with prednisolone resulted in better preserved bone mass. ${ }^{45}$ Corticosteroid-naïve patients treated with prednisolone lost more bone than those treated with budesonide, particularly during the first 6 months of treatment. In this study's patient group, a significant difference in change of bone mineral density between the treatment arms was evident from 6 months, when the percentage change from baseline was $-0.90 \%$ in the budesonide-treated patients versus $-3.35 \%$ in prednisolone-treated patients $(P=.002)$. These studies help illustrate how both decreasing systemic corticosteroids and reducing inflammation can have a positive impact on bone mineral density.

Both the AGA and BSG recommend keeping corticosteroid dosing to a minimum and advocate the early use of immunomodulators to help with this goal. In a study of 137 patients with 
inflammatory bowel disease, those in disease remission had higher age-matched bone mineral density scores (Z-scores) than those with active disease, and this had a correlation with length of remission. ${ }^{46}$ In fact, after 3 years of disease remission, bone mineral density approached normal when compared with age-matched control patients. Additionally, patients treated with azathioprine had significantly increased Z-scores, which correlated to the drug's ability to induce disease remission rather than a direct effect of the drug. The cumulative steroid dose was slightly higher in the group with active disease compared with the remission group. ${ }^{46}$

Does anti-TNF- $\alpha$ therapy positively affect bone mineral density and reduce fracture risk? There are a few studies examining this, but they are limited by small sample size or nonclinical endpoints. ${ }^{20,21,47}$ One retrospective study did show a greater increase in bone mineral density with the monoclonal anti-TNF- $\alpha$ antibody, infliximab concomitantly administered with bisphosphonates as compared with bisphosphonates alone; while infliximab alone failed to increase the bone mineral density. ${ }^{48}$ The authors speculated that a study with greater power might detect a significant increase in bone mineral density with anti-TNF- $\alpha$ alone. The mechanisms by which anti-TNF- $\alpha$ therapy could reduce bone loss in inflammatory bowel disease have not been investigated in humans. Anti-TNF- $\alpha$ treatment may provide direct protection against osteoclast-mediated bone destruction by depleting TNF- $\alpha$, a potent osteoclast activator. Alternatively, it might have a more indirect role by dialing down the inflammatory disease, reducing other osteoclast activators like IL-6 and IL-1, and improving the nutritional status of patient. We speculate that both indirect and direct mechanisms might play a role in preservation or increases in bone mineral density in treated patients. While the body of information is supportive of anti-TNF- $\alpha$ therapy, further studies with clinically relevant endpoints, including fracture reduction, are needed to elucidate its role in the management of osteoporosis in inflammatory bowel disease.

\section{CONCLUSION}

Osteoporosis imparts a significant burden on today's health care system, accounting for high costs, increased hospitalizations, disability, and time lost from work. Considerable information is known about osteoporosis and inflammatory bowel disease, but further work is needed. As more is understood about the pathophysiology linking these diseases, more treatment modalities will become available. For physicians, it is important to recognize the risk factors that are associated with inflammatory bowel disease and osteoporosis and screen the appropriate population of patients, which will hopefully lead to earlier recognition of disease and earlier, more appropriate preventive and treatment strategies.

\section{Acknowledgments}

Funding: Dr. Humphrey received funding from US Department of Veterans Affairs.

\section{References}

1. Assessment of fracture risk and its application to screening for post-menopausal osteoporosis. Report of a WHO Study Group. World Health Organ Tech Rep Ser 1994;843:1-129. [PubMed: 7941614]

2. Compston JE, Judd D, Crawley EO, et al. Osteoporosis in patients with inflammatory bowel disease. Gut 1987;28(4):410-415. [PubMed: 3583068]

3. Bjarnason I, Macpherson A, Mackintosh C, et al. Reduced bone density in patients with inflammatory bowel disease. Gut 1997;40(2):228-233. [PubMed: 9071937]

4. Pollak RD, Karmeli F, Eliakim R, et al. Femoral neck osteopenia in patients with inflammatory bowel disease. Am J Gastroenterol 1998;93(9):1483-1490. [PubMed: 9732930]

5. Bernstein CN, Leslie WD. The pathophysiology of bone disease in gastrointestinal disease. Eur J Gastroenterol Hepatol 2003;15(8):857-864. [PubMed: 12867794] 
6. Bernstein CN, Leslie WD, Leboff MS. AGA technical review on osteoporosis in gastrointestinal diseases. Gastroenterology 2003;124(3):795-841. [PubMed: 12612917]

7. Recommendations for the prevention and treatment of glucocorticoid-induced osteoporosis. American College of Rheumatology Task Force on Osteoporosis Guidelines. Arthritis Rheum 1996;39(11): 1791-1801. [PubMed: 8912500]

8. van Staa TP, Leufkens HG, Abenhaim L, et al. Oral corticosteroids and fracture risk: relationship to daily and cumulative doses. Rheumatology (Oxford) 2000;39(12):1383-1389. [PubMed: 11136882]

9. van Staa TP, Leufkens HG, Cooper C. The epidemiology of corticosteroid-induced osteoporosis: a meta-analysis. Osteoporos Int 2002;13(10):777-787. [PubMed: 12378366]

10. Neurath MF, Pettersson S. Predominant role of NF-kappa B p65 in the pathogenesis of chronic intestinal inflammation. Immunobiology 1997;198(1-3):91-98. [PubMed: 9442381]

11. Barnes PJ, Karin M. Nuclear factor-kappaB: a pivotal transcription factor in chronic inflammatory diseases. N Engl J Med 1997;336(15):1066-1071. [PubMed: 9091804]

12. Redlich K, Hayer S, Ricci R, et al. Osteoclasts are essential for TNF-alpha-mediated joint destruction. J Clin Invest 2002;110(10):1419-1427. [PubMed: 12438440]

13. Manolagas SC. The role of IL-6 type cytokines and their receptors in bone. Ann N Y Acad Sci 1998;840:194-204. [PubMed: 9629251]

14. Schulte CM, Dignass AU, Goebell H, et al. Genetic factors determine extent of bone loss in inflammatory bowel disease. Gastroenterology 2000;119(4):909-920. [PubMed: 11040178]

15. Boyce BF, Xing L. The RANKL/RANK/OPG pathway. Curr Osteoporos Rep 2007;5(3):98-104. [PubMed: 17925190]

16. Bernstein CN, Sargent M, Leslie WD. Serum osteoprotegerin is increased in Crohn's disease: a population-based case control study. Inflamm Bowel Dis 2005;11(4):325-330. [PubMed: 15803021]

17. Moschen AR, Kaser A, Enrich B, et al. The RANKL/OPG system is activated in inflammatory bowel disease and relates to the state of bone loss. Gut 2005;54(4):479-487. [PubMed: 15753532]

18. Hofbauer LC, Lacey DL, Dunstan CR, et al. Interleukin-1 beta and tumor necrosis factor-alpha, but not interleukin-6, stimulate osteoprotegerin ligand gene expression in human osteoblastic cells. Bone 1999;25(3):255-259. [PubMed: 10495128]

19. Miheller P, Muzes G, Racz K, et al. Changes of OPG and RANKL concentrations in Crohn's disease after infliximab therapy. Inflamm Bowel Dis 2007;13(11):1379-1384. [PubMed: 17663430]

20. Franchimont N, Putzeys V, Collette J, et al. Rapid improvement of bone metabolism after infliximab treatment in Crohn's disease. Aliment Pharmacol Ther 2004;20(6):607-614. [PubMed: 15352908]

21. Abreu MT, Geller JL, Vasiliauskas EA, et al. Treatment with infliximab is associated with increased markers of bone formation in patients with Crohn's disease. J Clin Gastroenterol 2006;40(1):55-63. [PubMed: 16340635]

22. Ducy P, Zhang R, Geoffroy V, et al. Osf2/Cbfa1: a transcriptional activator of osteoblast differentiation. Cell 1997;89(5):747-754. [PubMed: 9182762]

23. Gong Y, Slee RB, Fukai N, et al. LDL receptor-related protein 5 (LRP5) affects bone accrual and eye development. Cell 2001;107(4):513-523. [PubMed: 11719191]

24. Mbalaviele G, Sheikh S, Stains JP, et al. Beta-catenin and BMP-2 synergize to promote osteoblast differentiation and new bone formation. J Cell Biochem 2005;94(2):403-418. [PubMed: 15526274]

25. Ohnaka K, Tanabe M, Kawate H, et al. Glucocorticoid suppresses the canonical Wnt signal in cultured human osteoblasts. Biochem Biophys Res Commun 2005;329(1):177-181. [PubMed: 15721290]

26. Adams JS, Hewison M. Unexpected actions of vitamin D: new perspectives on the regulation of innate and adaptive immunity. Nat Clin Pract Endocrinol Metab 2008;4(2):80-90. [PubMed: 18212810]

27. Silvennoinen J, Lamberg-Allardt C, Karkkainen M, et al. Dietary calcium intake and its relation to bone mineral density in patients with inflammatory bowel disease. J Intern Med 1996;240(5):285292. [PubMed: 8946811]

28. Cashman KD, Shanahan F. Is nutrition an aetiological factor for inflammatory bowel disease? Eur J Gastroenterol Hepatol 2003;15(6):607-613. [PubMed: 12840670]

29. McCarthy D, Duggan P, O'Brien M, et al. Seasonality of vitamin D status and bone turnover in patients with Crohn's disease. Aliment Pharmacol Ther 2005;21(9):1073-1083. [PubMed: 15854168] 
30. Gilman J, Shanahan F, Cashman KD. Determinants of vitamin D status in adult Crohn's disease patients, with particular emphasis on supplemental vitamin D use. Eur J Clin Nutr 2006;60(7):889_ 896. [PubMed: 16493452]

31. Duggan P, O'Brien M, Kiely M, et al. Vitamin K status in patients with Crohn's disease and relationship to bone turnover. Am J Gastroenterol 2004;99(11):2178-2185. [PubMed: 15555000]

32. Szulc P, Chapuy MC, Meunier PJ, Delmas PD. Serum undercarboxylated osteocalcin is a marker of the risk of hip fracture: a three year follow-up study. Bone 1996;18(5):487-488. [PubMed: 8739908]

33. Watts NB, Lewiecki EM, Miller PD, Baim S. National Osteoporosis Foundation 2008 Clinician's Guide to Prevention and Treatment of Osteoporosis and the World Health Organization Fracture Risk Assessment Tool (FRAX): what they mean to the bone densitometrist and bone technologist. J Clin Densitom 2008;11(4):473-477. [PubMed: 18562228]

34. Kornbluth A, Hayes M, Feldman S, et al. Do guidelines matter? Implementation of the ACG and AGA osteoporosis screening guidelines in inflammatory bowel disease (IBD) patients who meet the guidelines' criteria. Am J Gastroenterol 2006;101(7):1546-1550. [PubMed: 16863559]

35. National Osteoporosis Foundation. Clinician's Guide to Prevention and Treatment of Osteoporosis. National Osteoporosis Foundation; Washington, DC: 2008.

36. Lewis, NR.; Scott, BB. Guidelines for Osteoporosis in Inflammatory Bowel Disease and Coeliac Disease. British Society of Gastroenterology; London: 2007.

37. Henderson S, Hoffman N, Prince R. A double-blind placebo-controlled study of the effects of the bisphosphonate risedronate on bone mass in patients with inflammatory bowel disease. Am J Gastroenterol 2006;101(1):119-123. [PubMed: 16405543]

38. Palomba S, Manguso F, Orio F Jr, et al. Effectiveness of risedronate in osteoporotic postmenopausal women with inflammatory bowel disease: a prospective, parallel, open-label, two-year extension study. Menopause 2008;15(4 Pt 1):730-736. [PubMed: 18698280]

39. Chestnut $\mathrm{CH}$ 3rd. Calcitonin in the prevention and treatment of osteoporosis. Osteoporos Int 1993;3 (Suppl 1):206-207. [PubMed: 8461562]

40. Rossouw JE, Anderson GL, Prentice RL, et al. Risks and benefits of estrogen plus progestin in healthy postmenopausal women: principal results from the Women's Health Initiative randomized controlled trial. JAMA 2002;288(3):321-333. [PubMed: 12117397]

41. Delmas PD, Bjarnason NH, Mitlak BH, et al. Effects of raloxifene on bone mineral density, serum cholesterol concentrations, and uterine endometrium in postmenopausal women. N Engl J Med 1997;337(23):1641-1647. [PubMed: 9385122]

42. Ettinger B, Black DM, Mitlak BH, et al. Reduction of vertebral fracture risk in postmenopausal women with osteoporosis treated with raloxifene: results from a 3-year randomized clinical trialMultiple Outcomes of Raloxifene Evaluation (MORE) Investigators. JAMA 1999;282(7):637-645. [PubMed: 10517716]

43. Neer RM, Arnaud CD, Zanchetta JR, et al. Effect of parathyroid hormone (1-34) on fractures and bone mineral density in postmenopausal women with osteoporosis. N Engl J Med 2001;344(19): 1434-1441. [PubMed: 11346808]

44. Vestergaard P, Rejnmark L, Mosekilde L. Fracture risk associated with different types of oral corticosteroids and effect of termination of corticosteroids on the risk of fractures. Calcif Tissue Int 2008;82(4):249-257. [PubMed: 18414920]

45. Schoon EJ, Bollani S, Mills PR, et al. Bone mineral density in relation to efficacy and side effects of budesonide and prednisolone in Crohn's disease. Clin Gastroenterol Hepatol Feb;2005 3(2):113-121. [PubMed: 15704045]

46. Reffitt DM, Meenan J, Sanderson JD, et al. Bone density improves with disease remission in patients with inflammatory bowel disease. Eur J Gastroenterol Hepatol 2003;15(12):1267-1273. [PubMed: 14624148]

47. Ryan BM, Russel MG, Schurgers L, et al. Effect of antitumour necrosis factor-alpha therapy on bone turnover in patients with active Crohn's disease: a prospective study. Aliment Pharmacol Ther 2004;20(8):851-857. [PubMed: 15479356]

48. Pazianas M, Rhim AD, Weinberg AM, et al. The effect of anti-TNF-alpha therapy on spinal bone mineral density in patients with Crohn's disease. Ann N Y Acad Sci 2006;1068:543-556. [PubMed: 16831950] 
Table 1

Risk Factors for Osteoporosis in Inflammatory Bowel Disease

Increasing age
Use of corticosteroids
Malnutrition
Low BMI
Malabsorption of vitamin D, calcium, and vitamin K
Immobilization
Previous fragility fracture
Hypogonadism
Smoking
Chronic inflammatory state including imbalance of the RANK-
RANKL OPG system

Abbreviations: $\mathrm{BMI}=$ body mass index RANK = receptor activator of NF- $\mathrm{kB} ; \mathrm{RANKL}=$ receptor activator of NF- $\mathrm{\kappa B}$ ligand; $\mathrm{OPG}=$ osteoprotegerin . 
Table 2

\title{
Treatment Options for Osteoporosis in Inflammatory Bowel Disease
}

\author{
Nonpharmacologic therapy \\ Regular weight-bearing exercise \\ Avoiding or quitting tobacco \\ Limited use of alcohol \\ Emphasis on better nutrition, particularly on vitamin D and calcium \\ Employment of fall prevention strategies \\ Pharmacologic therapy \\ Calcium and vitamin D supplementation \\ Bisphosphonates \\ Calcitonin \\ Cautious use of hormone replacement therapy for both women and men \\ Recombinant parathyroid hormone (teriparatide) \\ Minimizing corticosteroid use with the early use of immunomodulating agents
}

